\title{
TRATAMENTO ENDODÔNTICO EM DENTE DESVITALIZADO POR TRAUMA: RELATO DE CASO CLÍNICO
}

\section{ENDODONTIC TREATMENT IN DEVITALIZED TRAUMATIZED TOOTH: A CASE REPORT}

\author{
Gabriela Granata Astolfi* \\ Marlowa Marcelino Crema* \\ Priscyla Waleska Simões"** \\ Renan Antonio Ceretta***
}

\begin{abstract}
RESUMO
O objetivo deste trabalho é apresentar um relato de caso clínico de uma paciente com necessidade de tratamento endodôntico no elemento 41, com necropulpectomia decorrente de trauma de luxação. Paciente de 12 anos, gênero feminino, leucoderma, procurou atendimento na Clínica Universitária para tratar o dente traumatizado. Em exames clínicos e radiográficos, observou-se necrose e escurecimento coronário do elemento 41 . Foi realizado tratamento endodôntico em duas sessões. O elemento foi instrumentado com sistema manual de limas até a K\#50, irrigando-se com hipoclorito de sódio a 1\% alternadamente com cada lima. Após o procedimento, foi realizada a irrigação final com ácido etilenodiamino tetra-acético a 17\% e hipoclorito de sódio a $1 \%$, e a secagem com pontas de papel estéreis. A obturação foi feita pela técnica de compactação lateral mais McSppaden (Ténica Híbrida de Tagger), utilizando-se cones de guta percha e cimento de óxido de zinco e eugenol. O caso está sendo proservado há 8 meses, sem sintomatologia, apresentando repleção endodôntica do conduto radicular com normalidade em região apical e redução do escurecimento coronário, segundo imagem periapical e acompanhamento radiográfico.
\end{abstract}

Descritores: Tratamento do canal radicular • Cavidade pulpar • Traumatismos dentários • Necrose da polpa dentária.

\section{ABSTRACT}

The aim of this study is to present a case report of a patient in need of endodontic treatment in the element 41 , with necropulpectomy due to trauma. The patient was 12 years old, female, white skin, and she sought treatment at the University Odontologic Service to treat a traumatized tooth. In clinical and radiographic examination, there was necrosis and coronary darkening of the element 41. Endodontic treatment was conducted in two sessions. The element was instrumented with manual files system until $\mathrm{K} \# 50$, irrigating the canal with sodium hypochlorite $1 \%$ alternating with each file. After the procedure, the final irrigation was performed with ethylenediamine tetraacetic acid $17 \%$ and $1 \%$ sodium hypochlorite and drying with sterile paper points. The obturation was made by lateral compaction technique more McSppaden (Tagger Hybrid Technique), using gutta percha points and cement zinc oxide and eugenol. The case is being following 8 months without symptoms, with repletion of the endodontic root canal, normally in the apical region and reduction in coronary darkening, according periapical image and radiographic follow-up.

Descriptors: Root canal therapy $\bullet$ Dental pulp cavity $\bullet$ Tooth injuries $\bullet$ Dental pulp necrosis

\footnotetext{
* Graduanda em Odontologia - Universidade do Extremo Sul Catarinense - Email: gabigastolfi@gmail.com.

** Graduação pela UFSC/SC. Especialista em Endodontia UFSC/SC. Mestre em Endodontia pela São Leopoldo Mandic/SP. Coordenadora das disciplinas de Endodontia e Clínica Integrada da Universidade do Extremo Sul Catarinense. E-mail: marlowacrema@gmail.com.

*** Doutora em Ciências da Saúde - Universidade do Extremo Sul Catarinense. Docente do Curso de Odontologia e no Programa de Pós-Graduação em Saúde Coletiva (PPGSCol) - Universidade do Extremo Sul Catarinense, Criciúma, SC, Brasil. Docente do Centro de Engenharia, Modelagem e Ciências Sociais Aplicadas (CECS), Universidade Federal do ABC (UFABC), São Bernardo do Campo, SP, Brasil. Email: pritsimoes@gmail.com.

**** Doutor em Ciências da Saúde. Professor e coordenador do Curso de Odontologia - Universidade do Extremo Sul Catarinense. Email: rce@unesc.net.
} 


\section{N T R O D U ÇÃ O}

O traumatismo dental está cada vez mais presente no cotidiano da população devido ao aumento da violência, acidentes de trânsito e a prática de esportes radicais ${ }^{1,2,3,4}$.

Estudos $^{1-5}$ sobre a prevalência de traumatismos e injúrias dentárias relataram que o trauma acomete mais o sexo masculino em relação ao feminino e tem o incisivo central superior como o dente mais afetado.

A perda de estrutura e função dos elementos dentais, decorrentes do trauma, pode prejudicar a qualidade de vida em crianças e adolescentes ${ }^{6}$. Uma pesquisa mostrou que crianças que sofreram injúrias devido ao trauma, tiveram dificuldade ao sorrir e preocupação com o que os outros pensam sobre seus dentes anteriores fraturados ou restaurados ${ }^{7}$.

Algumas sequelas possíveis do trauma dentoalveolar são a obliteração do canal pulpar e a reabsorção radicular inflamatória. Devido a isso, a busca pelo tratamento após o trauma e o acompanhamento do cirurgião-dentista são necessários para que o diagnóstico e o tratamento adequado sejam realizados com o intuito de prevenir demais danos $^{8}$.

Dentre os traumatismos, a luxação é uma das modalidades traumáticas que acarretam os tecidos moles. Esse dano ao ligamento periodontal é caracterizado pelo deslocamento do dente no sentido lingual/palatal ou vestibular. Esse dente deve ser reposicionado e acompanhado clínica e radiograficamente. Caso apresente sinais de necrose pulpar como escurecimento da coroa, resposta negativa ao teste de vitalidade pulpar, sinais radiográficos de lesões periapicais, o tratamento endodôntico é recomendado para evitar a ocorrência de reabsorção externa da raiz ${ }^{9}$.

Mediante o exposto, este relato de caso clínico teve como objetivo analisar a perda de vitalidade de um dente devido à luxação, mostrando as consequências desse tipo de acidente em pré-adolescentes e a conduta correta para o seu tratamento, através da avaliação clínica e radiográfica.

\section{REVIS ÃO B I BLIOGRÁF I C A}

A luxação é um traumatismo de maior intensidade aos tecidos moles, caracterizado pelo deslocamento dental nos sentidos palatino, vestibular, mesial ou distal. Clinicamente o dente pode apresentar mobilidade ou não, presença de sangramento e lacerações. O tratamento vai depender do grau de magnitude do deslocamento e mobilidade, podendo ser utilizadas contenções flexíveis ou semirrígidas ${ }^{10}$. Um estudo realizado em 2010 sobre o tratamento de urgências em crianças de até 12 anos constatou que a luxação é segunda injúria mais prevalente $(19,21 \%)$, antecedida apenas pelas fraturas coronárias $(53,78 \%)^{11}$.

Sobre as técnicas de instrumentação dos canais radiculares, um estudo prospectivo de coorte realizado em 2011, avaliou o tratamento endodôntico de 816 dentes com instrumentação endodôntica convencional, obteve, após 5 anos de acompanhamento, $92.3 \%$ de sucesso nos canais tratados com necrose pulpar sem lesões periapicais ${ }^{12}$.

Uma pesquisa realizada em 2010, para comparar a eficácia da instrumentação manual com limas-K e a instrumentação de sistema rotatório em canais em forma de "C" utilizando 24 molares mandibulares, demonstrou que a instrumentação rotatória ProTaper manteve as curvaturas do canal, instrumentando com rapidez e poucos erros e a instrumentação manual obteve uma limpeza eficaz ${ }^{13}$.

Em 2007, um estudo com o intuito de comparar a qualidade da modelagem final de canais radiculares achatados utilizou 12 caninos humanos com as técnicas de instrumentação Rotatória Profile Maillefer e Crown Down manual. Após análise em microscópio óptico, os autores, Cerqueira et $a .{ }^{14}{ }^{\text {, chegaram à conclusão de que não }}$ houve diferença significativa entre as técnicas e, mesmo que ambas não conseguissem preparar completamente as paredes dos canais, obtiveram um bom desempenho ${ }^{14}$.

Já em outra pesquisa feita em 2015, sobre a eficácia da modelagem dos canais
ASTOLFI GG

CREMA MM

SIMÕES PW

CERETTA RA

tratamento

ENDODONTICO

EM DENTE

DESVITALIZADO POR

tRAUMA: RELATO DE

caso cLÍNICO

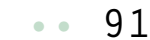

REV, ODONTOL.

UNIV, CID, SÃo

PAULO

2016; 29(1): $90-9$ JAN - ABR 
ASTOLFI GG

CREMA MM

SIMÕES PW

CERETTA RA

TRATAMENTO

ENDODONTICO

EM DENTE

DESVITALIZADO POR

TRAUMA: RELATO DE

CASO CLINICO

\section{2}

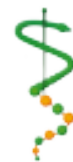

REV, ODONTOL

UNIV, CID, SÃO

PAULO

$2016 ; 29(1): 90-9$,

$J A N-A B R$ radiculares das limas rotatórias ProTaper e limas-K de aço inoxidável testadas em 60 molares maxilares, foi demonstrado que o sistema ProTaper instrumentou mais faces do que a técnica manual, porém nenhuma das técnicas conseguiu preparar o canal completamente ${ }^{15}$.

A associação da instrumentação biomecânica, que remove os detritos suspensos nas paredes pulpares, com a irrigação, responsável pela dissolução de restos de tecidos no canal pulpar e ação antibacteriana, é essencial para se obter um adequado debridamento do canal radicular ${ }^{16}$, 17.

O hipoclorito de sódio $(\mathrm{NaOCl})$ tem sido o irrigante de primeira escolha devido a sua capacidade de dissolver tecidos e por ter efeito antibacteriano, especialmente contra o biofilme ${ }^{16}$. Entretanto, ele é extremamente cáustico quando em contato com tecidos vitais. Uma revisão sistemática de 2013, que analisou as publicações sobre a extrusão dos irrigantes durante procedimentos endodônticos e seus fatores causantes, concluiu que as causas mais citadas na literatura foram tipo e profundidade de inserção da agulha, instrumentação exacerbada, perfuração, lesões ósseas nos casos em que ocorre diminuição da resistência dos tecidos periapicais e alto fluxo da irrigação ${ }^{16}$.

Em endodontia, o hipoclorito de sódio pode ser usado em diferentes concentrações: $0,5 \%, 1 \%, 2,5 \%, 5,25 \%$. A ação antibacteriana está relacionada à capacidade de oxidar e hidrolisar células proteicas bacterianas e, como esse efeito depende da quantidade de cloro livre disponível por meio da reação com substâncias inorgânicas, uma irrigação frequente em concentrações mais baixas pode ter o mesmo resultado do uso de concentrações mais elevadas. Portanto, a concentração de $\mathrm{Na}$ OCl para irrigação endodôntica pode ser de $0,5 \%$ a $1 \%$ com pH próximo da neutralidade, possuindo eficácia antimicrobiana e mínimo efeito irritante aos tecidos ${ }^{18,19}$.

Uma pesquisa realizada em 2001, sobre os efeitos do $\mathrm{NaOCl}$ em componentes sanguíneos e sangramento simulado, concluiu que os componentes da hemoglobina se decompõem para produzir ferro. Este é subsequentemente transformado no pigmento biliverdina que também pode causar descoloração do substrato do dente se não for removido ${ }^{17}$.

O ácido etilenodiamino tetra-acético (EDTA) é um quelante usado principalmente durante a irrigação final em conjunto com o hipoclorito de sódio com o intuito de remover a smear layer e auxiliar na limpeza e desinfecção das paredes dentinárias, além de ajudar na identificação de canais calcificados ${ }^{18,20}$. A ação resulta em paredes mais limpas, o que permite uma melhor atividade do hipoclorito de sódio durante a irrigação ${ }^{20}$.

A limpeza dos canais conseguida pela instrumentação associada a substâncias químicas deve ser mantida através do selamento temporário para se evitar uma reinfecção dos canais radiculares entre as sessões e após o tratamento ${ }^{21}$. O selamento provisório tem a finalidade de impedir a entrada de fluidos e bactérias provenientes da cavidade bucal nos canais. Para isso, esse material deve ter uma boa resistência à abrasão e compressão e um bom selamento hermético periférico ${ }^{22}$. Alguns materiais utilizados são o IRM, cimento de ionômero de vidro, cimento de óxido de zinco e eugenol, resinas fotopolimerizáveis $^{21}$.

Dentre os medicamentos intracanais, o hidróxido de cálcio tem sido considerado o melhor medicamento de escolha devido a sua capacidade de estimular a deposição de tecido duro e promover a cicatrização de polpas vitais e tecidos periapicais $^{23}$. Uma revisão de literatura de 1999, sobre as formulações do hidróxido de cálcio e seus melhores veículos, mostrou que o veículo desempenha o papel mais importante no processo, pois ele determina a velocidade de dissociação iônica e faz com que a pasta possa ser solubilizada e reabsorvida em várias taxas pelos tecidos periapicais. Os veículos viscosos e oleosos são os que mais prolongam a ação do hidróxido de cálcio em comparação com substâncias solúveis em água ${ }^{23}$.

Uma meta-análise, feita em 2007, sobre o efeito antibacteriano do hidróxido de cálcio como medicamento intracanal, concluiu que o hidróxido de cálcio tem eficácia limitada na eliminação de bactérias do canal radicular quando avaliado 
por técnicas de cultura ${ }^{24}$.

Para comparar o efeito de nanopartículas de óxido de cálcio e de hidróxido de cálcio sobre a bactéria Enterococcus faecalis, um estudo em 2016 utilizou modelos de dentina para a realização dos testes. Conforme os resultados, as nanopartículas de hidróxido de cálcio se mostraram mais eficientes do que as nanopartículas de óxido de cálcio, sendo um potente medicamento intracanal ${ }^{25}$.

Com o intuito de comparar as atividades antibacterianas do hidróxido de cálcio, hidróxido de cálcio misturado com o zeólito de prata e hidróxido de cálcio misturado com $2 \%$ de clorexidina na eliminação de $E$. faecalis, um estudo in vitro de 2016 chegou ao resultado de que a mistura de zeólito de prata com hidróxido de cálcio aumenta a atividade antibacteriana $^{26}$.

Dentre as diversas técnicas de obturação dos canais radiculares, a técnica híbrida de Tagger consiste na associação da condensação lateral ativa no terço apical com a compactação termomecânica feita com McSpadden ${ }^{27}$. Uma pesquisa de 2006, realizada na Faculdade de Odontologia da UFBA, comparou o selamento apical em dentes obturados pelas técnicas da condensação lateral, híbrida de Tagger e Thermafil. As três técnicas de obturação analisadas apresentaram algum grau de infiltração apical, não havendo diferença estatística significativa entre elas ${ }^{28}$.

Uma pesquisa feita em 2005 analisou, por meio de exames radiográficos, a qualidade do preenchimento de canais laterais de quatro técnicas diferentes: condensação lateral, híbrida de Tagger, McSpadden e Thermafil usando o cimento Grossman. A partir dos resultados obtidos, chegou-se à conclusão de que as técnicas que utilizavam a guta-percha termoplastificada tiveram melhor desempenho do que a técnica de condensação lateral, sendo a técnica do sistema Thermafil superior à híbrida de Tagger e à McSpadden, sendo estas últimas semelhantes entre $\mathrm{si}^{29}$.

Para analisar os efeitos de diferentes técnicas de obturação na resistência de união de cimentos à base de resina epóxi, um estudo de 2016 utilizou 60 caninos mandibulares para os testes. Concluiu que a técnica de condensação lateral teve maior força de união do que a técnica híbrida de Tagger, seguida pela técnica de cone único com a menor força de união ${ }^{30}$.

\section{RELATO DE CASO}

Relato de caso, no qual os dados da paciente foram retirados do prontuário individual, fornecido pela Clínica Odontológica da Universidade do Extremo Sul Catarinense (UNESC), da cidade de Criciúma-SC. A pesquisa foi aprovada pelo Comitê de Ética em seres humanos do local de origem sob o protocolo 1.367.468/2015, a carta de aceite foi assinada pela coordenadora da clínica de odontologia e o termo de consentimento livre e esclarecido foi assinado pelo responsável da paciente, contendo no prontuário, além de dados da paciente, os tratamentos já realizados e laudos radiográficos e fotográficos do caso.

Paciente, sexo feminino, 12 anos de idade, alérgica à amoxicilina, procurou atendimento na clínica odontológica da Universidade do Extremo Sul Catarinense, relatando o escurecimento de um dente inferior. Pelo relato constatou-se que esse dente havia sofrido trauma há um ano, e que foi procurado atendimento odontológico emergencial onde o cirurgião-dentista o reposicionou, pois estava vestibularizado, porém sem apresentar alterações no exame radiográfico periapical na época.

Foram realizados na UNESC, pelo acadêmico responsável do caso, anamnese, exame extra e intraoral, exames radiográficos e teste de vitalidade pulpar no elemento 41 com spray a $-40^{\circ}$ (Endo-Frost Spray, Roeko). Através desses exames, constatou-se que o elemento 41 apresentava um acinzentamento da coroa, sem sintomatologia e sem alterações radiográficas, conforme a Figura 1, porém com resultado negativo ao teste de vitalidade, comprovando necrose pulpar decorrente do trauma, classificado como luxação. O elemento com necropulpectomia foi submetido ao tratamento endodôntico na Clínica Universitária.

O tratamento endodôntico foi realizado em duas sessões. Na primeira consulta foram realizados anamnese, exames clíni-
ASTOLFI GG

CREMA MM

SIMÕES PW

CERETTA RA

TRATAMENTO

ENDODONTICO

EM DENTE

DESVITALIZADO POR

tRAUMA: RELATO DE

caso cLÍNICO

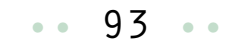

REV, ODONTOL.

UNIV, CID, SÃo

PAULO

2016; 29(1): $90-9$ JAN - ABR 
ASTOLFI GG

CREMA MM

SIMÕES PW :

CERETTA RA

TRATAMENTO

ENDODONTICO

EM DENTE

DESVITALIZADO POR

TRAUMA : RELATO DE

CASO CLINICO

$\therefore 94 \ldots$

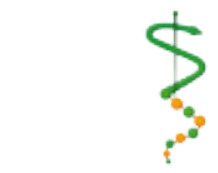

REV, ODONTOL

UNIV, CID, SÃO

PAULO

2016; 29(1): 90-9, JAN - ABR
ISSN 1983-5183

cos extra e intraorais, radiografia inicial, abertura coronária, isolamento absoluto, contorno e desgaste compensatório, exploração do canal e sua odontometria, instrumentação com limas Kerr e cinemática de $1 / 4$ de volta em vai e vem com técnica escalonada, sendo que o Comprimento Real do Dente é de $21 \mathrm{~mm}$; Comprimento de Trabalho: 20mm; Lima Patência: \#10; Lima Anatômica: \#20; Lima Memória: \#35, seguindo com irrigação abundante com hipoclorito de sódio a $1 \%$ a cada troca de lima. Para uma melhor finalização da instrumentação do canal, ao final do uso das limas, foram utilizadas brocas Gates \#4 e \#3 e limas Hedstroen \#40, \#45 e \#50.

Após, foi realizada irrigação final com $3 \mathrm{~mL}$ de ácido etilenodiamino tetra-acético (EDTA) a 17\% e hipoclorito de sódio a $1 \%$, secagem do canal radiculares com cones de papel absorvente (Tanari Industrial, Amazônia), medicação intracanal com Ultra-Cal XS (Ultradent) e selamento da coroa com ionômero de vidro (MaxxionR, FGM). As radiografias periapicais da odontometria e instrumentação estão demonstradas na Figura 2.
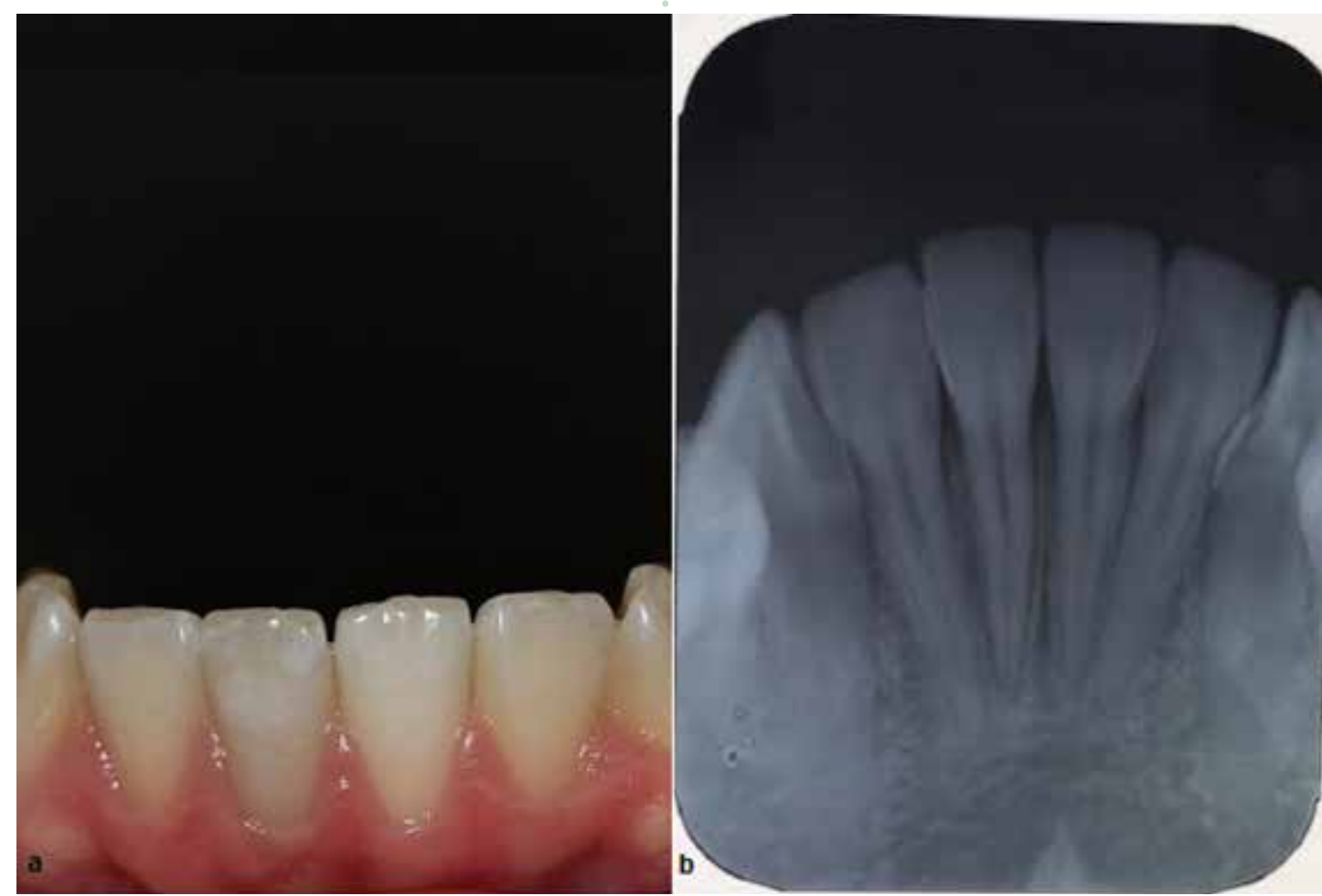

Figura 1 A: Foto extraoral demonstrando o acinzentamento da coroa do elemento 41. B: Radiografia periapical inicial.
Na segunda consulta foi realizada a revisão da anamnese, isolamento absoluto, remoção do hidróxido de cálcio, irrigação final com $3 \mathrm{~mL}$ de EDTA a $17 \%$ e hipoclorito de sódio a $1 \%$, secagem do canal com cones de papel absorvíveis (Tanari Industrial, Amazônia), prova do cone principal, obturação pela Técnica Híbrida de Tagger, cone de guta-percha calibrado em lima memória \#35 e cones acessórios B7 e B8 (Tanari Industrial, Amazônia) com o cimento obturador de óxido de zinco e eugenol (Biodinâmica, Paraná), seguido de restauração definitiva com resina composta e radiografia periapical do tratamento.

Não foi realizado nenhum procedimento estético clareador no elemento dental.

Após 8 meses de acompanhamento, o elemento 41 permanece sem sintomatologia, com replecão do conduto radicular e normalidade em região apical segundo as radiografias periapicais e redução do acinzentamento coronário segundo acompanhamento fotográfico, como demonstra a Figura 3. 

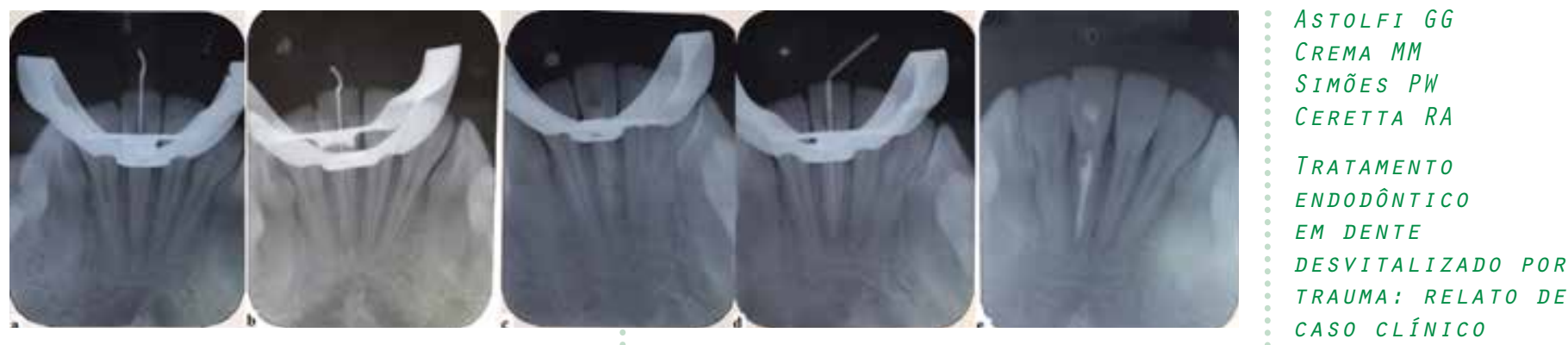

Figura 2. A: Odontometria com lima K\#10 no limite apical. B: Lima anatômica K\#20 no comprimento de trabalho. C: Medicação intracanal com Ultra-Cal XS. D: Conemetria utilizando cone principal de guta-percha \#35. E: Radiografia periapical para confirmação da obturação.

\section{I SCUSSÃO}

O objetivo principal do estudo foi analisar a perda de vitalidade de um dente devido à luxação por meio de um relato de caso realizado na clínica odontológica da Universidade do Extremo Sul Catarinense. Através da avaliação clínica e radiográfica e do tratamento adequado indicado, o caso permanece com normalidade do tratamento endodôntico após o acompanhamento de 8 meses. Comparando os resultados com os dados obtidos na literatura, nossos achados são semelhantes aos encontrados em 2011, quando a taxa de sucesso do tratamento endodôntico convencional em dentes com necrose pulpar

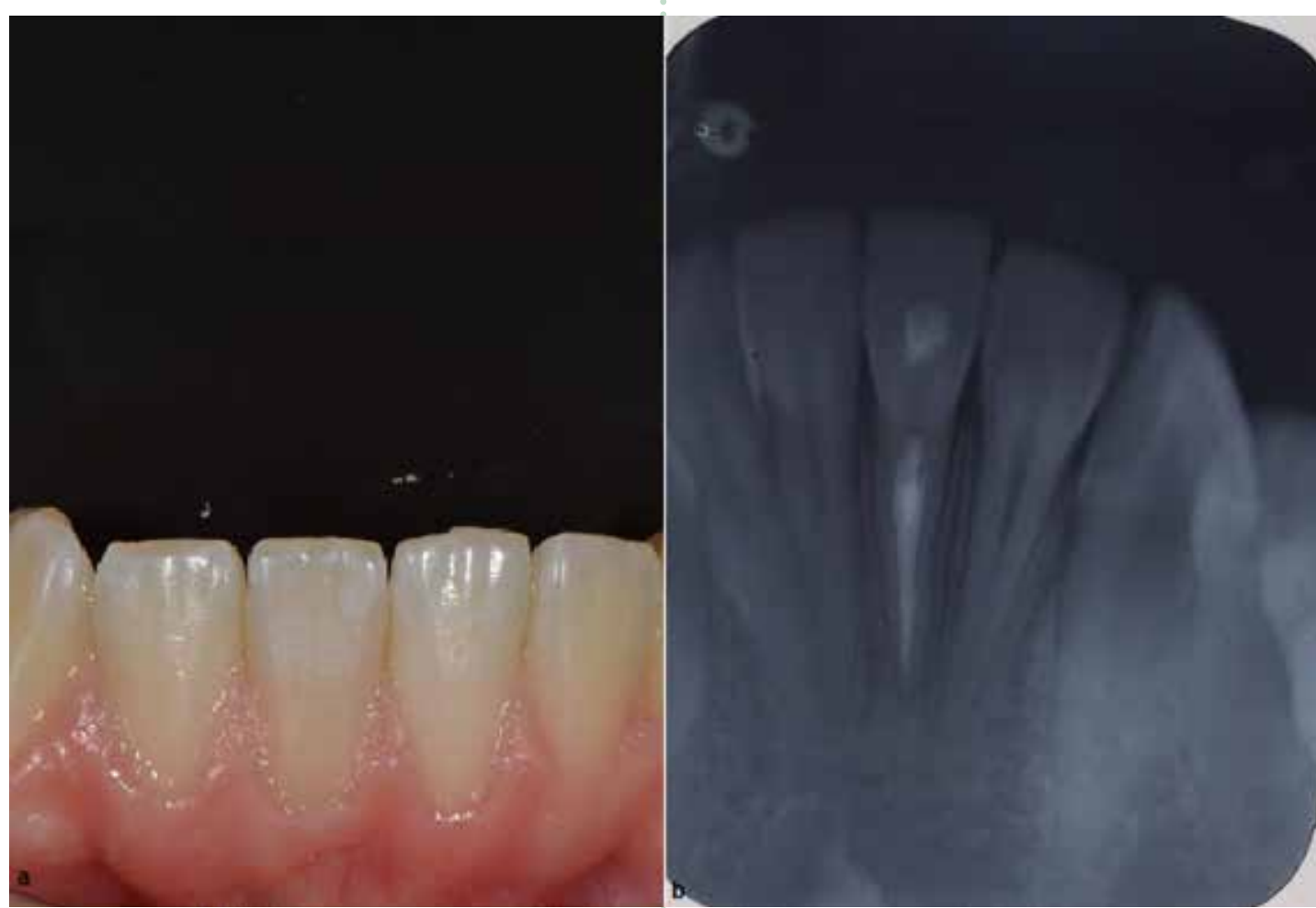

Figura 3. A: Foto extraoral demonstrando a diminuição do acinzentamento da coroa do elemento 41. B: Radiografia periapical no oitavo mês de acompanhamento. sem lesões periapicais foi de $92.3 \%{ }^{12}$.

Pesquisas comparando a técnica manual com limas-K e a técnica rotatória chegaram à conclusão de que o sistema ProTaper instrumentou mais faces do que a técnica manual, porém nenhuma das técnicas conseguiu preparar o canal completamente ${ }^{15}$. Em canais em forma de " $\mathrm{C}$ ", a técnica rotatória manteve as curvaturas do canal, instrumentando com rapidez e poucos erros e a instrumentação manual obteve uma limpeza eficaz ${ }^{13}$. Já em canais radiculares achatados, não houve diferença significativa entre as técnicas e, mesmo que ambas não conseguissem preparar completamente as paredes dos canais, ob-

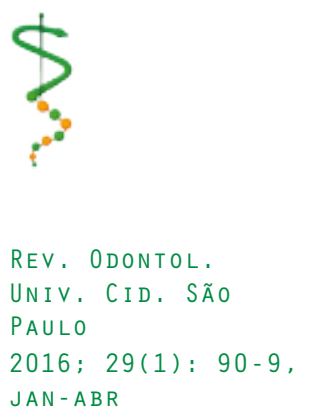


ASTOLFI GG

CREMA MM

SIMÕES PW

CERETTA RA

TRATAMENTO

ENDODONTICO

EM DENTE

DESVITALIZADO POR

TRAUMA: RELATO DE

CASO CLINICO

96

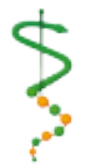

REV, ODONTOL.

UNIV, CID, SÃO

PAULO

2016; 29(1): $90-9$,

$J A N-A B R$ tiveram um bom desempenho ${ }^{14}$. A técnica utilizada no caso foi a manual com limas-K que, conforme a literatura, são eficazes em sua limpeza e possuem bom desempenho.

O adequado debridamento do canal radicular é feito através da utilização da instrumentação biomecânica, com o intuito de entrar em contato e remover todos os detritos suspensos nas paredes pulpares e espaços dos canais laterais, associado à irrigação. A irrigação tem parte essencial para a remoção desses detritos, possuindo efeitos mecânicos, que são o desprendimento e remoção dos microrganismos, restos de tecido pulpar, detritos de dentina e produtos da instrumentação. Seus efeitos químicos são a dissolução de restos de tecidos, detritos de dentina, smear layer, perturbação química de biofilmes, morte de microrganismos e inativação de seus subprodutos $^{16,17}$.

A irrigação do canal radicular do caso foi feita com hipoclorito de sódio a $1 \%$ pois, conforme a literatura, os estudos ${ }^{16-19}$ observados concordaram em que o hipoclorito de sódio é o irrigante mais usado durante a instrumentação dos canais radiculares, devido à sua capacidade de dissolver tecidos e ao efeito antibacteriano, sendo efetivo durante a limpeza e desinfecção dos canais radiculares. Pode ser utilizado em várias concentrações em endodontia, mas sempre tomando-se os cuidados necessários para evitar seu extravasamento devido à sua toxicidade ${ }^{16-19}$.

A concentração mais indicada para dentes necrosados é de $2,5 \%$ a $5,25 \%^{31}$, porém a utilizada no caso foi de $1 \%$, por se tratar de um tratamento realizado por estudantes, devido ao menor efeito irritante aos tecidos, caso haja extravasamento ${ }^{17}$, 18 .

Outras importantes características do hipoclorito de sódio são os efeitos clareador, saponificador, desnaturador de proteínas, tensoativo, umectante, desodorizante $^{32}$. O seu efeito clareador juntamente com a remoção dos detritos e restos de tecidos pulpares necrosados podem explicar a diminuição do acinzentamento coronário do elemento afetado do nosso caso clínico.

A associação do ácido etilenodiami- no tetraacético ao hipoclorito de sódio durante a irrigação final mostra-se essencial para se alcançar uma limpeza do canal superior, resultando em uma melhor adaptação dos materiais obturadores e medicação intracanal ${ }^{20}$. Mesmo possuindo fraco efeito antibacteriano e antifúngi$\mathrm{CO}^{18}$, a capacidade do EDTA em reduzir a extensão de smear layer, melhora a habilidade e penetração do hipoclorito de sódio na dentina, aumentando o efeito antibacteriano do $\mathrm{NaOCl}^{20}$.

Como medicação intracanal, o hidróxido de cálcio, composto por pó, um veículo e um radiopacificador, é considerado o melhor medicamento para a deposição de tecido duro e promoção da cicatrização de polpas vitais e tecidos periapicais ${ }^{23}$, sendo o material de escolha para a medicação intracanal do caso.

Para combater o biofilme de E. faecalis, alguns autores ${ }^{25}$ constataram que as nanopartículas de hidróxido de cálcio são mais eficientes do que outros materiais intracanais. Acrescentando a este estudo, Ghatole e colaboradores concluíram que a mistura de zeólito de prata com hidróxido de cálcio apresenta maior atividade antibacteriana contra $E$. faecalis ${ }^{26}$. Já para Sathorn, Parashos e Messer, o hidróxido de cálcio possui eficácia limitada na eliminação de bactérias, quando avaliado através de culturas ${ }^{24}$.

Existem diversas técnicas de obturação dos canais radiculares, entre elas a condensação lateral, híbrida de Tagger ${ }^{27}$ e cone único e Thermafil. Segundo estu$\operatorname{dos}^{28-30}$ que avaliaram a eficácia dessas técnicas, Carvalho et al. ${ }^{28}$ (2006) validaram que todas demonstraram algum grau de infiltração apical ${ }^{28}$. Sobre a força de união, Rached-Júnior et al. ${ }^{30}$ (2016) chegaram à conclusão de que a condensação lateral foi superior à técnica híbrida de Tagger $^{30}$. Contrastando a isso, Raymundo et al. ${ }^{29}$ (2005) mostraram que as técnicas que utilizavam a guta-percha termoplastificada obtiveram melhor desempenho do que a técnica de condensação lateral ${ }^{29}$. Conforme o exposto, percebe-se que cada técnica possui suas vantagens e desvantagens. A técnica utilizada em nosso caso clínico foi a híbrida de Tagger, por ser mais acessível e de baixo custo. 
O caso permanece sem sintomatologia, com repleção do conduto radicular, normalidade em região apical, segundo as radiografias periapicais e redução do acinzentamento coronário, segundo acompanhamento fotográfico.

\section{CONCLUSÕES}

A partir do exposto, o correto tratamento para dentes que sofreram trauma de luxação seria o cauteloso acompanhamento clínico e radiográfico do caso.

As técnicas e materiais utilizados mostraram-se eficazes em todas as fases do tratamento.
Mesmo sem procedimentos clareadores estéticos, a limpeza do canal com a instrumentação manual e irrigação com hipoclorito de sódio conseguiu obter um bom resultado no clareamento coronário do elemento afetado.

Embora os resultados do estudo sejam importantes, uma possível limitação seria o pequeno tamanho da amostra, por se tratar do relato de um único caso, sendo recomendada a realização de mais estudos com um maior número de casos sobre o efeito clareador do hipoclorito de sódio em elementos com escurecimento coronário, decorrentes de necrose pulpar.

\section{REFERENNCIAS}

1. Traebert J, Almeida ICS, Garghetti C, Marcenes W. Prevalência, necessidade de tratamento e fatores predisponentes do traumatismo na dentição permanente de escolares de 11 a 13 anos de idade. Cad Saúde Pública 2004 abr;20(2):403-10.

2. Traebert J, Marcon KB, Lacerda JT. [Prevalence of traumatic dental injuries and associated factors in schoolchildren of Palhoca, Santa Catarina State]. Ciencia \& saude coletiva 2010 Jun;15 Suppl 1(1849-55.

3. Souza-Filho FJ, Soares AJ, Gomes BPFA, Zaia AA, Ferraz CCR, Almeida JFA. Avaliação das injúrias dentárias observadas no Centro de Trauma Dental da Faculdade de Odontologia de Piracicaba-Unicamp. RFO 2010 maio-ago;14(2):111-6.

4. Pedroni LBG, Barcellos LA, Miotto MHMB. Tratamento em dentes permanentes traumatizados. Pesqui bras odontopediatria clín integr 2009 jan-abr ;9(1):107-12.

5. Skaare $A B$, Jacobsen I. Dental injuries in Norwegians aged 7-18 years. Dental traumatology:official publication of International Association for Dental Traumatology 2003 Apr;19(2):6771.
6. Antunes LA, Leao AT, Maia LC. [The impact of dental trauma on quality of life of children and adolescents: a critical review and measurement instruments]. Ciencia \& saude coletiva 2012 Dec;1 7(12):3417-24.

7. Bendo CB, Paiva SM, Torres CS, Oliveira AC, Goursand D, Pordeus IA, et al. Association between treated/untreated traumatic dental injuries and impact on quality of life of Brazilian schoolchildren. Health and quality of life outcomes 2010 Oct 04;8(114.

8. Andreasen FM, Kahler B. Pulpal response after acute dental injury in the permanent dentition: clinical implications - a review. Journal of endodontiCs 2015 Mar;41(3):299-308.

9. Diangelis AJ, Andreasen JO, Ebelese$\operatorname{der} K A$, Kenny DJ, Trope $M$, Sigurdsson $A$, et al. International Association of Dental Traumatology guidelines for the management of traumatic dental injuries: 1. Fractures and luxations of permanent teeth. Dental traumatology:official publication of International Association for Dental Traumatology 2012 Feb;28(1):2-12.

10. Losso EM, Tavares MCR, Bertoli FMP, Baratto-Filho F. Traumatismo dentoalveolar na dentição decídua. SBO 2011 jan-mar;8(1):114-6.
ASTOLFI GG

CREMA MM

SIMÕES PW

CERETTA RA

TRATAMENTO

ENDODONTICO

EM DENTE

DESVITALIZADO POR

tRAUMA: RELATO DE

caso CLÍNICO
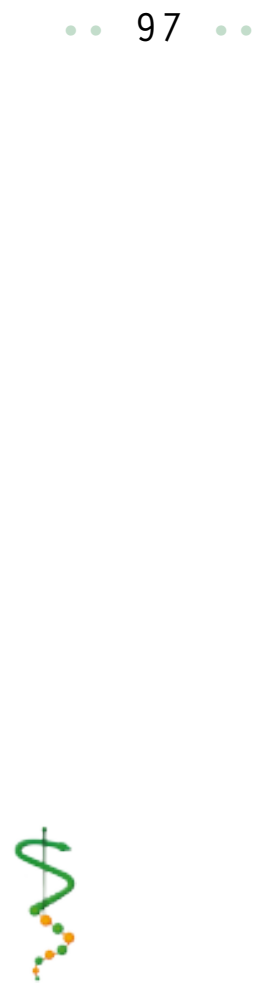

REV, ODONTOL.

UNIV, CID, São

PAULO

2016; 29(1): 90-9, JAN - ABR 
ASTOLFI GG

CREMA MM :

SIMÕES PW

CERETTA RA

TRATAMENTO

ENDODONTICO

EM DENTE

DESVITALIZADO POR

TRAUMA : RELATO DE

CASO CLINICO

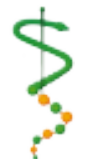

REV, ODONTOL

UNIV, CID, SÃO

PAULO

$2016 ; 29(1): 90-9$,

$J A N-A B R$
11. Paschoal MAB, Gurgel CV, Lourenço Neto N, Kobayashi TY, Silva SMB, Abdo RCC, et al. Perfil de tratamento de urgência de crianças de 0 a 12 anos de idade, atendidas no Serviço de Urgência Odontológica da Faculdade de Odontologia de Bauru da Universidade de São Paulo. Odontol Clín-Cient 2010 abr;9(3):243-7.

12. Ricucci D, Russo J, Rutberg M, Burleson JA, Spangberg LS. A prospective cohort study of endodontic treatments of 1,369 root canals: results after 5 years. Oral surgery, oral medicine, oral pathology, oral radiology, and endodontics 2011 Dec;112(6):825-42.

13. Yin $X$, Cheung GS, Zhang C, Masuda YM, Kimura Y, Matsumoto K. Micro-computed tomographic comparison of nickel-titanium rotary versus traditional instruments in C-shaped root canal system. Journal of endodontics 2010 Apr;36(4):708-12.

14. Cerqueira LG, Gomes CC, Penina P, Prado MA, Freitas LF, Camões ICG, et al. Técnicas de instrumentação manual e rotatória: comparação da modelagem dos canais radiculares. UFES Rev Odontol 2007 jan-abr;9(1):13-9.

15. Stavileci M, Hoxha V, Gorduysus O, Tatar I, Laperre K, Hostens J, et al. Evaluation of Root Canal Preparation Using Rotary System and Hand Instruments Assessed by Micro-Computed Tomography. Medical science monitor basic research 2015 Jun 20;21(123-30.

16. Boutsioukis C, Psimma Z, Van Der Sluis LW. Factors affecting irrigant extrusion during root canal irrigation: a systematic review. International endodontic journal 2013 Jul;46(7):599618.

17. Yamaguchi H, Hosoya N, Kobayashi K, Yokota T, Arai T, Nakamura J, et al. The influence of two concentrations of sodium hypochlorite on human blood: changes in haemolysis, $\mathrm{pH}$ and protein. International endodontic journal 2001 Apr;34(3):231-6.
18. Hauman $\mathrm{CH}$, Love RM. Biocompatibility of dental materials used in contemporary endodontic therapy: a review. Part 1. Intracanal drugs and substances. International endodontic journal 2003 Feb;36(2):75-85.

19. Dakin HD. On the use of certain antiseptic substances in the treatment of infected wounds. British medical journal 1915 Aug;2(2852):318-20.

20. Hulsmann M, Heckendorff M, Lennon $\mathrm{A}$. Chelating agents in root canal treatment: mode of action and indications for their use. International endodontic journal 2003 Dec;36(12):81030 .

21. Couto LHA, Pinheiro JMM, Couto PHA, Freitas MRLS. Avaliação in vitro da microinfiltração coronária em cinco materiais seladores temporários usados em endodontia. Arqu bras odontol 2010 6(2):78-88.

22. Nabeshima CK, Britto MLB. Avaliação da resistência do Cimpat ${ }^{\circledR}$ rosa como restauração temporária durante tratamento endodôntico. IJD Int J Dent 2008 jul-set;7(3):158-61.

23. Fava LR, Saunders WP. Calcium hydroxide pastes: classification and clinical indications. International endodontic journal1999 Aug;32(4):25782.

24. Sathorn C, Parashos P, Messer H. Antibacterial efficacy of calcium hydroxide intracanal dressing: a systematic review and meta-analysis. International endodontic journal 2007 Jan;40(1):2-10.

25. Louwakul P, Saelo A, Khemaleelakul S. Efficacy of calcium oxide and calcium hydroxide nanoparticles on the elimination of Enterococcus faecalis in human root dentin. Clinical oral investigations 2017 Apr;21(3):865-71. 
26. Ghatole K, Gowdra RH, Azher S, Sabharwal S, Singh VT, Sundararajan BV. Enhancing the antibacterial activity of the gold standard intracanal medicament with incorporation of silver zeolite: An in vitro study. Journal of International Society of Preventive \& Community Dentistry 2016 Jan-Feb;6(1):75-9.

27. Tagger M, Tamse A, Katz A, Korzen $\mathrm{BH}$. Evaluation of the apical seal produced by a hybrid root canal filling method, combining lateral condensation and thermatic compaction. Journal of endodontics 1984 Jul;10(7):299-303.

28. Carvalho E, Júnior JA, Malvar MF, Albergaria S. Avaliação do selamento apical em dentes obturados pela técnica da condensação lateral híbrida, de Tagger e Thermafil. Rev Cienc méd biol 2006 set-dez;5(3):239-44.
29. Raymundo A, Leonardi DP, Portela CP, Baratto Filho F. Radiographic analysis of the lateral canals filling by four different obturation techniques. RSBO (Impr);2(2), nov 20052005 nov ;2(2):23-7.

30. Rached-Júnior FJA, Souza AM, Macedo LMD, Raucci-Neto W, Baratto-Filho F, Silva BM, et al. Effect of root canal filling techniques on the bond strength of epoxy resin-based sealers. Braz oral res 2016 abr;30(1):1-5.

31. Pretel H, Bezzon F, Faleiros FBC, Dametto FR, Vaz LG. Comparação entre soluções irrigadoras na endodontia: clorexidina $\mathrm{x}$ hipoclorito de sódio. RGO Rev Gaucha Odontol (Online) 2011 jun;v. 59(supp.):127-32.

32. Fabro RMN, Britto MLB, Nabeshima CK. Comparação de diferentes concentrações de hipoclorito de sódio e soro fisiológico utilizados como soluções irrigadoras Odontol clín-cient 2010 out-dez;9(4):365-8.

Recebido em 10/10/2016

Aceito em 08/12/2016
ASTOLFI GG

CREMA MM

SIMÕES PW

CERETTA RA

tratamento

ENDODONTICO

EM DENTE

DESVITALIZADO POR

TRAUMA: RELATO DE

caso cLÍnICO

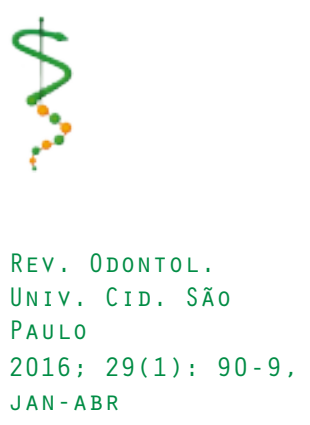

